\title{
Social Media Use and Exposure to Health Related False Information
}

\author{
Tandiyo Pradekso ${ }^{1}$, S. Rouli Manalu ${ }^{2}$, Djoko Setyabudi ${ }^{3}$ \\ \{tandiyop@yahoo.com $\left.{ }^{1}\right\}$ \\ Universitas Diponegoro, Indonesia ${ }^{1,2,3}$
}

\begin{abstract}
Social media have largely been associated to the propelling distribution of misinformation, disinformation, false information, and few other terms alike. The study explores the sort of health-related false information that are usually sought through the social media by most people, and how they are related to the types of social media used. There is a rising concern about the quality and validity of health information online, which is not infrequently contain misleading content and pose high risk to susceptible users of social media. The findings revealed that television is still the most used medium, yet the portion of social media usage is closing to the television. Regarding the circulation of health information, the main three types of social media (Instagram, Facebook, WhatsApp) share an approximate portion as health information sources. Certain health-related false information issue is somewhat associated to certain type of social media use.
\end{abstract}

Keywords: Social-Media, False-Information.

\section{Introduction}

Social media have largely been associated to the propelling distribution of misinformation, disinformation, false information, and few other terms alike [1][2]. Some scholars have made observation that social media, have become the sources of information where people go to find relevant knowledge when they encounter health problems. Not only because many people share their experiences, stories, and have discussion about health-related issues in social media, but also because many healthcare facilities, and even medical doctors have increasing present on social media as a channel to deliver their health information.

In contrast, there is a rising concern about the quality and validity of health information online, which is not infrequently contain misleading content and pose high risk to susceptible users of social media. The case in point is health information on social media in Indonesia. IDN Times mentioned that $95 \%$ of health information circulated through WhatsApp application are wrong information or false information [3].

The recent practice of disseminating information is characterized with peer-to-peer sharing, as well as by sharing the content across different social media types. The information supply is increasing exponentially since readers can also be producers of information by the practice of sharing [4][5][6]. A study of Giglietto et al [7], states that the decision to disseminate information from the public interest often arises from motivation to inform a novelty to others and to protect the interests of the public. In cases where there is false news spread by someone with consideration of the audience, often this aims to influence others to 
have the same view with him/her, or to support and raise an issue that will benefit the interests of the news disseminator. However, public interest is not the only consideration for disseminating information. There are also individuals who disseminate information for his/her own interest [7]. Often there are cases arise when someone disseminates information for personal gain, and for the purpose of gaining fame or to be considered relevant in the dynamics of society.

\section{Research Method}

This paper explores the sort of health-related false information that are usually sought through the social media by most people, and how they are related to the types of social media used, and it was based on the data of a study that was carried out in field survey. The analysis is based on data that was collected for health-related survey in East Java in 2019. The research population is residents of the province of East Java, aged 17-65 years old. Sampling was defined in multi-stage random sampling, starting from the municipality, followed by subdistrict, by village and finally selected at neighborhood (RW/RT) level. With $95 \%$ level of confidence, the sample from the province is 400 respondents. Cross sectional explanations are carried out to seek out variables involved in the issue.

\section{Result and Discussion}

\subsection{The Media Habit}

The media consumption among respondents of East Java shows that television (mostly broadcast or free to air broadcasting) is the most used medium in their daily life. Nevertheless, the share of social media (mostly Facebook, Instagram, Twitter, WhatsApp, and YouTube) usage is closing to the television. The other available media such as print media (newspaper, magazine, tabloid), radio, and online media comprises only four percent of total media consumption as respondents' main source of information. The large slice of social media (see Figure 1) as the most used medium indicates a significant shift to the new media consumption from the traditional mass media routine. Among the uses of social media, WhatsApp is apparently the most used social medium. WhatsApp is primarily used by two-third of all respondents who have social media (54\%), followed by Facebook, and Instagram subsequently. Other social media that served as the main medium, has only four percent's share and consist of Line, YouTube, and Twitter.

The data took a different shape when it comes to the most trusted medium. The credibility of social media was declining more than a half compare to its usage. Whilst the credibility's of television and other traditional mass media were high. Print media and online media are trusted three times and six times higher equate to their usage. 


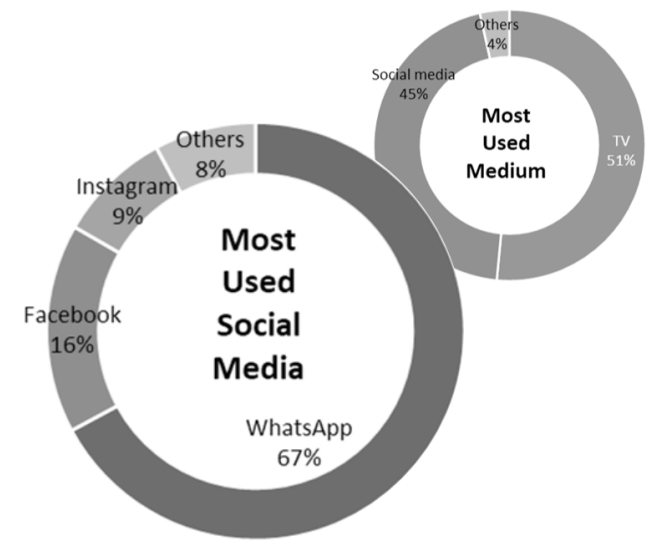

Fig. 1. Most used medium and social media.

A reshaping proportion also appeared to the trustworthiness of social media. Just like television, WhatsApp is still considered to have high credibility by most of the respondents. However, the share decreases about thirty percent relative to its usage. On the other hand, the share of all other social media is increasing. Especially the Instagram that doubled the share when it gets to the most trusted social medium (see Figure 2).

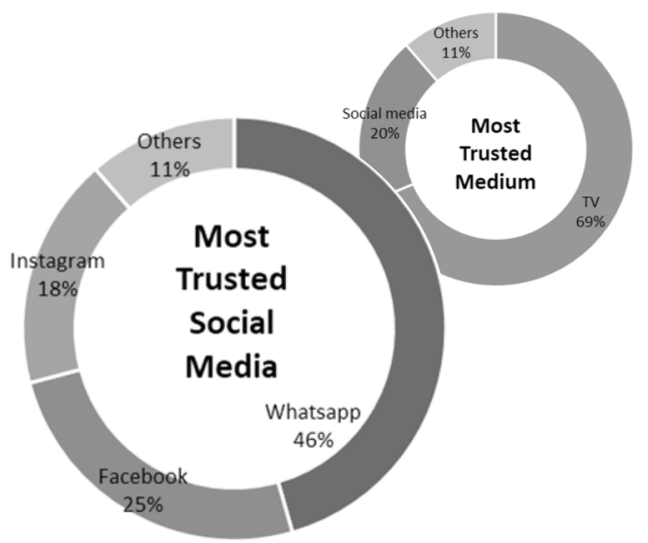

Fig. 2. Most trusted medium and social media.

\subsection{Health Information Sources}

There are two opposing facts arise in relation to circulation of health-related information on the Internet and social media. One side of the coin shows that posting, commenting, discussing, and disseminating health information on social media and the Internet can lead to the emergence of heath communities online that drive increasing access to health information. Sharing information based on patients' experiences is also seen as contextualizing medical information that can benefited other patients that come to the same health problems. This type of information is valued as experiential knowledge about health. Communicating health- 
related issues among patients, or laypeople, has been considered as a way for health empowerment in the society. However, another side of the coin shows that there is a risk in trusting health information online, since not all health-related information that can be found on social media and the Internet is valid and contain accurate and verifiable information. Media reports show a disturbing fact about high number of health related false information that are spreading through social media applications. Even though in many cases the information transfers are without malicious intent, the health information online still poses some risks to

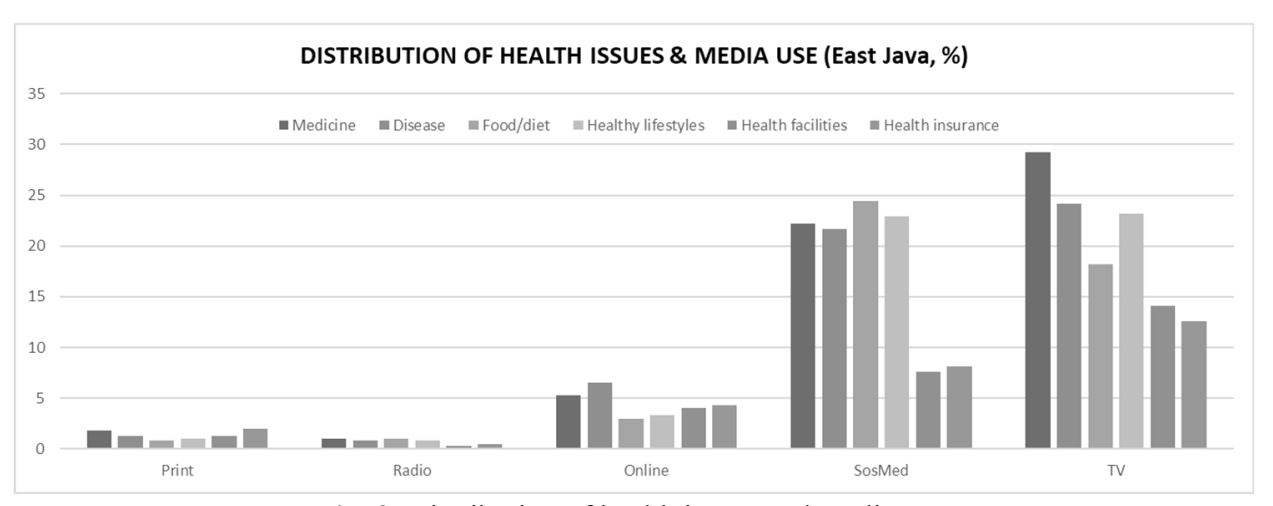

Fig. 3. Distribution of health issues and media use. susceptible Internet users.

The health information exercised in the study are divided into six broad general categories of medicines, diseases, food/diet/nutrition, healthy lifestyles, health facilities, and health insurance. The distribution profile of health issues are fairly similar between television and social media (see Figure 3). For the more essential issues such as medicine, diseases, health facilities, and health insurance, television share the bigger portions, where as in social media food, diet, and nutrition leads the segments.

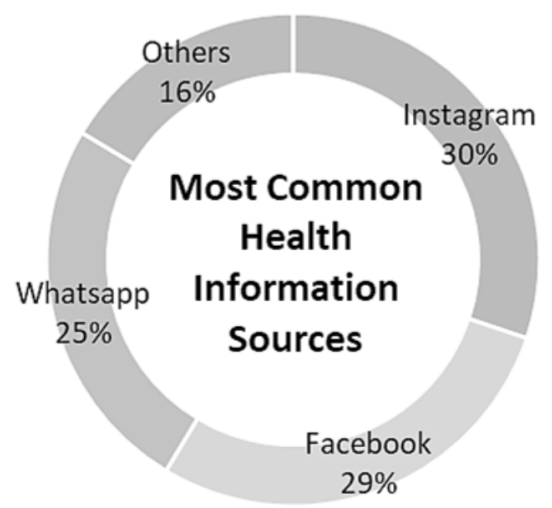

Fig. 4. Most common health information social media sources.

Regarding the circulation of health information, the main three types of social media share an approximate portion as health information sources. However, the distribution of health-related information circulation across the three main types of social media used reveals that Instagram and Facebook are surpassing WhatsApp as the source of health information. It 
is reasonable though since WhatsApp was basically a chatting application, before advances also as social medium (see Figure 4). A detail examination on the use of social media in East Java disclosed that WhatsApp is mostly used as the medium of communication and information sharing. For information seeking, respondents of East Java use typically use Facebook and Instagram instead of WhatsApp.

The use of Instagram and Facebook as the health information sources are related to the age of the respondents. The fact that Instagram is more popular among the younger age is reflected in its use as the main health information source. The use of Instagram is negatively related to age, it is decreasing with the increasing of age. Conversely, the use of Facebook is increasing along with the increasing of age. The trend of WhatsApp is similar to Facebook, and finally WhatsApp dominates the most common health information source among the respondents of 50 years of age and above.

\subsection{Health-related False Information}

The current practice of disseminating information is characterized with the repetition of peer-to-peer sharing, as well as by sharing content among different platforms of social media. The ease of information spreading by copying hyperlinks over social media is a common practice. News readers can also be news distributors. The emergence of new media is increasingly becoming a reference because it is considered more independent and does not have economic interests that must be protected. But this, in turn, generates a new problem, because at the same time there will be sources of information that appear to be reliable but are actually disseminate information that cannot be justified. This circumstance raises the amount of information that contradicts one another and requires the reader to be able to digest and compare information.

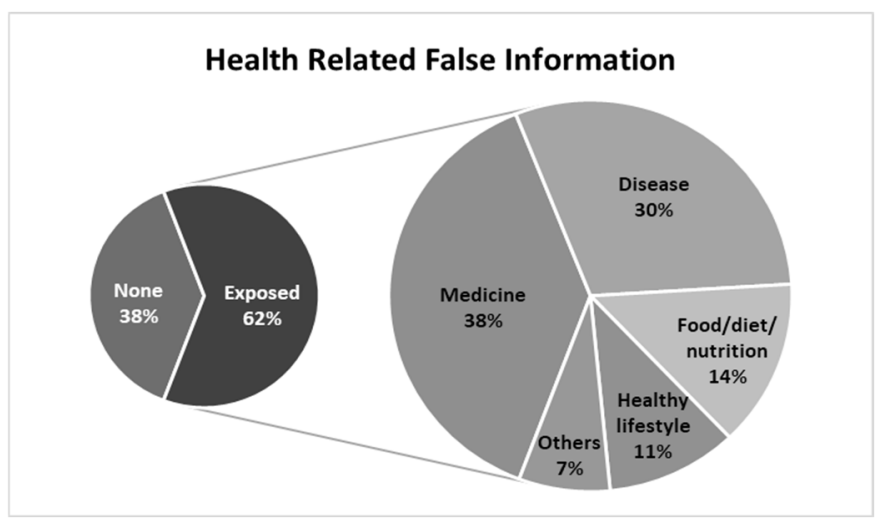

Fig. 5. Health-related false information.

In the case of health-related false information, most of the respondents stated that they have the experience of exposed to those kinds of information. And out of 62 percent who were exposed to health-related false information, most of them were exposed to medicine and disease issues (Figure 5). The fact that the respondents could point out health-related false information based on their experiences tells that to some extents there are awareness of the spreading of that types of information. This also demonstrated that respondents are able to digest and compare information. 


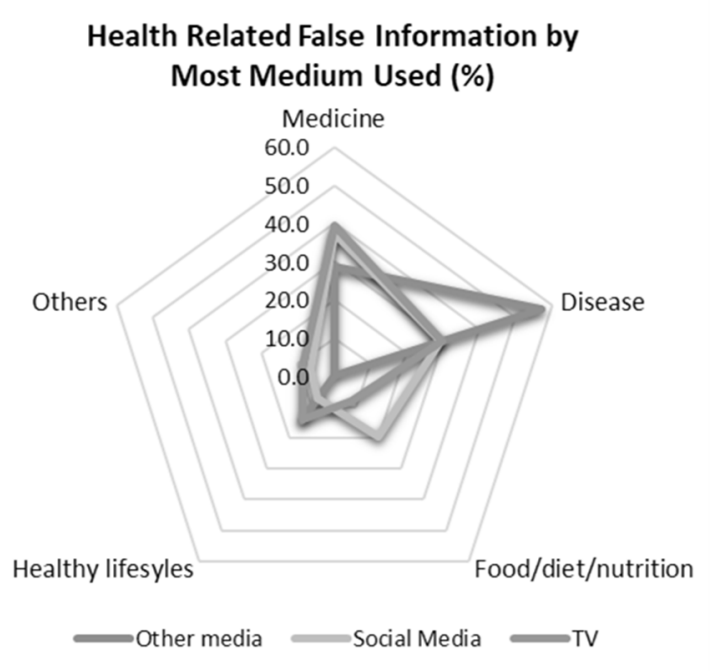

Fig. 6. Health-related false information by most medium used.

A cross-section analysis of health-related false information, based on the most medium used reveals that television and social media use share the same pattern of dominant healthrelated false issues with a minor exception in healthy lifestyles and diet issues. Television and social media display a relatively high portion of false information exposure about medicine. They also share the same moderate exposure of false information about disease. The difference between the two laid on the issue of food/diet/nutrition and healthy lifestyles (see Figure 6). Health-related false information about healthy lifestyles are frequently discovered in television compared to social media. In contrast, false information on food/diet/nutrition appeared more in social media. False information about disease is considered to appear more in other media, a composite of online media, print media, and radio, than television and social media.

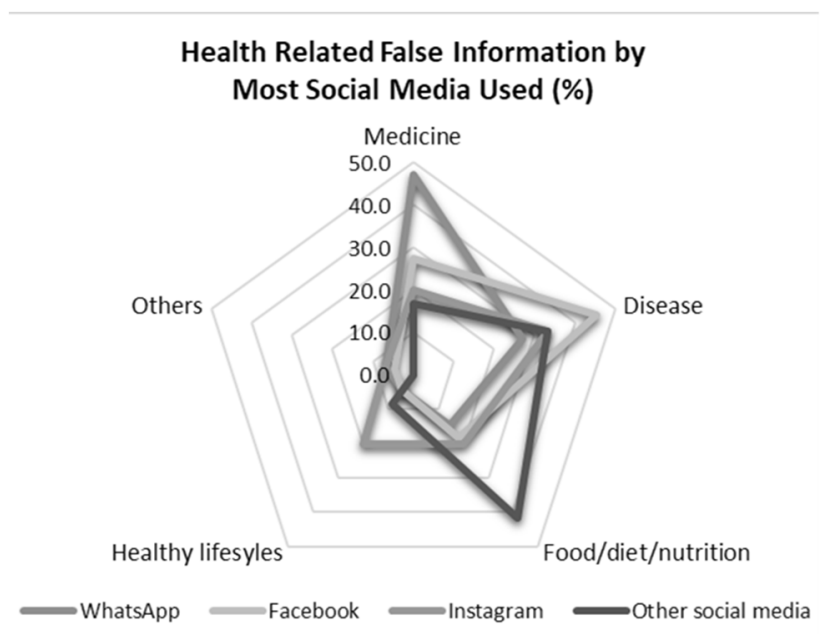

Fig. 7. Health-related false information by most social media 
Among the most social media used, the distribution frequent exposure of health-related false information is uniquely tied to the typical platform of social media. There is a different pattern for each type of social media use on the dominant issue of health-related false information. Medicine-related false information is perceived predominantly in WhatsApp. Disease-related false information is largely found in Facebook, while healthy lifestyles-related false information mostly found in Instagram. Health-related false information about food, diet, and nutrition frequently found in other social media, a combination of YouTube, Line, and Twitter.

\section{Conclusion}

The cross-sectional analysis of types of health-related false information, most medium used, and most social media used reveals some noteworthy points. Regarding the most social media used, certain health-related false information issue is somewhat associated to certain type of social media use. Whilst, social media and television use share the same pattern to certain types of health-related false information.

\section{References}

[1] L. Bode and E. K. Vraga, "In related news, that was wrong: The correction of misinformation through related stories functionality in social media," J. Commun., vol. 65, no. 4, pp. 619-638, 2015.

[2] G. S. O'Keeffe and K. Clarke-Pearson, "The impact of social media on children, adolescents, and families," Pediatrics, vol. 127, no. 4, pp. 800-804, 2011.

[3] M. J. Times, I. D. N., \& Damanik, "Ketua Dewan Pers: 95 Persen Informasi Kesehatan di Whatsapp Hoax." [Online]. Available: https://www.idntimes.com/news/indonesia/margith-juitadamanik/ketuadewan-pers-95-persen-informasi-kesehatan-di-whatsapp-hoax. [Accessed: 27Feb-2019].

[4] A. Hermida, "From TV to Twitter: How ambient news became ambient journalism," Media/Culture J., vol. 13, no. 2, 2010.

[5] H. Li and Y. Sakamoto, "Computing the veracity of information through crowds: a method for reducing the spread of false messages on social media," in 2015 48th Hawaii International Conference on System Sciences, 2015, pp. 2003-2012.

[6] J. Lee and Y. Choi, "Informed public against false rumor in the social media era: Focusing on social media dependency," Telemat. Informatics, vol. 35, no. 5, pp. 1071-1081, 2018.

[7] F. Giglietto, L. Iannelli, L. Rossi, and A. Valeriani, "Fakes, news and the election: A new taxonomy for the study of misleading information within the hybrid media system," Convegno AssoComPol, 2016. 